\title{
An Efficient Routing Protocol Design for Distributed Wireless Sensor Networks
}

\author{
Prof.K. Manikandan \\ School of Computing Science and Engineering \\ VIT University \\ Vellore - 632014 \\ Tamilnadu, India
}

\author{
Dr.T.Purusothaman \\ Dept of CSE \& IT \\ Government College of Technology \\ Coimbatore-641 013. \\ Tamilnadu, India
}

\begin{abstract}
Wireless sensor networks (WSN) represent a new paradigm shift in Adhoc networks. Wireless Sensor Networks consists of small nodes with sensing, computation and communication capabilities. The sensor networks task is to sense the environment, and relay the information back to a remote base station, where the user can access it. Sensor nodes are deployed in a monitoring region to gather information on environments like temperature, humidity, position, sound etc. Many routing protocols have been specifically designed for Wireless Sensor Networks where energy awareness is an essential design issue. Overall, the routing techniques are classified into three categories based on the underlying network structure: flat, hierarchical and location based routing. Energy Conservation is the major issue in distributed wireless sensor network. A new approach of Optimal Energy Efficient Clustering Algorithm (OEECA) is proposed in this paper. Also, we compare OEECA (proposed approach) with LEACH (Low Energy Adaptive Cluster Head) Routing Algorithm. Comparison is based on Energy consumption ,Selection of Cluster-Head node and Sending data's to the Base-station. From this Comparison study, we predict that,OEECA algorithm is better in the case of consume less energy by the cluster-nodes, selection of Cluster-head and sending more data's consumes less energy as efficient than LEACH.
\end{abstract}

\section{Keywords}

Sensor networks, routing protocol, energy, network structure, monitoring region

\section{Introduction}

Sensor networking is a challenging research area that draws on contributions from signal processing, networking and protocols, databases and information management, distributed algorithms, embedded systems, and architecture and QoS In Recent years, Wireless sensor networks becomes the most interesting networking technologies to provide the sensed aggregated data to the Base station with limited power capability. These networks consist of a large number of sensor nodes densely distributed over the region of interest for collecting information or monitor \& track certain specific phenomena from the physical environment.WSNs are likely to operate under very dynamic and critical environment with applications such as environmental monitoring, public safety, medical, transportation and military. Moreover, sensor nodes use broadcast communication with each other.

Wireless communication endowed with numerous advantages over traditional wired network and enables to develop small, low-cost, low power and multi-functional sensing devices. These small sensing devices have the capabilities of sensing, computation, self organizing and communication known as sensors. Sensor is a tiny device used to sense the ambient condition of its surroundings, gather data, and process it to draw some meaningful information which can be used to recognize the phenomena around its environment. These sensors can be grouped together using mesh networking protocols to form a network communicating wirelessly using radio frequency channel. The collection of these homogenous or heterogeneous sensor nodes called wireless sensor network (WSN) [1]. The ability of low cost, small size and easy deployment of the sensor nodes make it possible to deploy them in a large number in an area to be investigated [2]. Interestingly, unlike other networks that performs poor with growth in their networks size, WSN get stronger and performs better as much as number of nodes exceeds. In addition, without any complexity in configuration network size can be extended simply by adding additional number of nodes. Therefore, it is said that connectivity using mesh networking will occupy any possible communication path in search of destination using node to node hoping. Owing all these considerable advantages, application domain of WSNs varies from environmental monitoring, to health care applications, military operation, to transportation, to security applications, to weather forecasting, to real time tracking [7].

In this paper, OEECA (Proposed algorithm) to compare with LEACH routing algorithm. In OEECA algorithm, the secondary cluster-head can be selected to minimize the searching over-head and with limited amount of energy more data have to be send. As in the LEACH routing algorithm, randomized cluster-head can be selected and it consumes more energy to send data to base station as compared to OEECA algorithm. 


\section{SENSOR SYSTEM ARCHITECTURE}

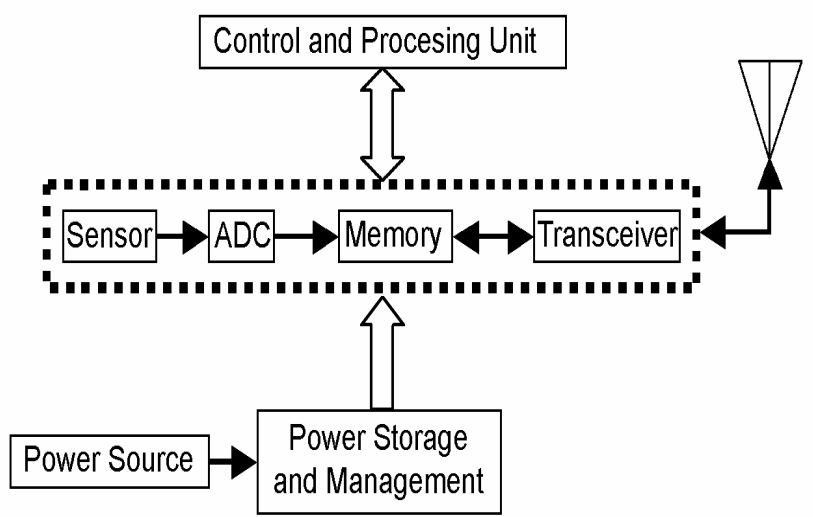

Fig 2.1: Sensor system Architecture

A sensor node is an entity that includes a sensor, power source, memory, communication module, processing and control unit. Sensor have a transducer that converts a physical event into electrical or other signals that can be read by an observer or by a device.

\subsection{Sensor node}

Also known as a mote, this is the basic unit in a sensor network and is capable of performing some processing, gathering sensory information, and communicating with other connected nodes in the network.

\subsection{Memory Unit}

This unit of sensor node is used to store both the data and program code. In order to store data packets from neighboring nodes Random Only Memory (ROM) is normally used. And to store the program code, flash memory or Electrically Erasable Programmable Read Only Memory (EEPRM) is used.

\subsection{Power Unit}

For computation and data transmission, the corresponding units in sensor node need power (energy). A node consist a power unit responsible to deliver power to all its units. The basic power consumption at node is due to computation and transmission where transmission is the most expensive activity at sensor node interms of power consumption.

\subsection{Processing Unit}

Sensor node has a microcontroller which consist a processing unit, memory, converters (analog to digital) timer and Universal Asynchronous Receive and Transmit (UART) interfaces to do the processing tasks. This unit is responsible for data acquisition, processing incoming and outgoing information, implementing and adjusting routing considering the performance conditions of the transmission.

\subsection{Communication Unit}

Senor nodes use radio frequencies or optical communication in order to achieve networking. This task is managed by radio units in sensor nodes that use electromagnetic spectrum to convey the information to their destinations. Usually each sensor node transfers the data to other node or sinks directly or via multi hop routing. Sensor nodes use broadcast communication with each other, while in ad-hoc networks[1,2]

\section{LITERATURE SURVEY \& RELATED WORKS}

\subsection{Taxonomy of Routing Protocols}

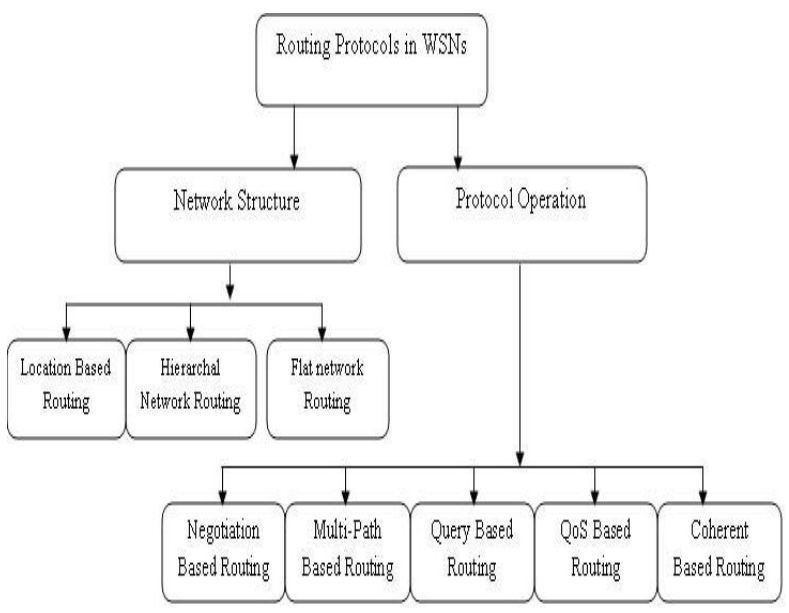

Fig 3.1: Routing protocols in WSN

\subsection{Clustering Algorithm}

Clustering is especially important for sensor network applications. In cluster networks, sensors are partitioned in to smaller clusters and cluster head $(\mathrm{CH})$ for each cluster is elected. Sensor nodes in each cluster transmit their data to the respective cluster head $(\mathrm{CH})$ and $\mathrm{CH}$ aggregates data and forward them to a central base station. Clustering through creating a hierarchical WSN facilitates efficient utilization of limited energy of sensor nodes and hence extends network lifetime[8].

Sensor nodes in clusters transmit messages over a short distance (within clusters), more energy is drained from $\mathrm{CHs}$ due to message transmission over long distances $(\mathrm{CHs}$ to the base Station) compared to other sensor nodes in the cluster. Periodic re-election of $\mathrm{CHs}$ within clusters based on their residual energy is a possible solution to balance the power consumption of each cluster. Clustering Approach leads to form group of clusters inside the network to communicating with each other. The cluster-head is selected to send the aggregated data to the basestation[7] .

The Selection of Cluster-head is based on the Clustering algorithm approaches are LEACH (Low Energy Adaptive Clustering Hierarchy).This Clustering concepts 
deals with the selection of cluster-head, formation of clusters, selection of cluster-head for next round and eligibility for cluster head selection. The following Clustering module forms six groups with their respective Cluster-heads,

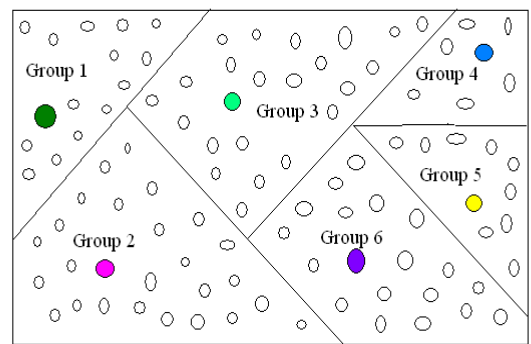

Shaded colours are cluster heads $(\mathrm{CH})$ and various nodes are grouped as clusters

\section{Fig3.2: Clustering form}

3.3 Research on Energy Efficient communication protocol for wireless micro sensor networks by W.Heinemann, A. Chandrakasan,H. Balakrishnan proposed an solution that,

LEACH [2,3] routing protocol is to efficiently maintain the energy consumption and performing data aggregation

in order to reduce the number transmitted messages to the sink. The operation of LEACH is divided in two phases,

(i) Set phase.

(ii) Steady state phase.

\subsubsection{Set-up phase}

In set-up phase, the cluster head is selected and then it forms a group.So after some time the corresponding cluster head energy to be reduced and to rotate the cluster head selection process. The cluster head selection is based on which the threshold $\mathrm{T}(\mathrm{s})$,

\section{$\mathrm{T}(\mathrm{s})=\left\{\operatorname{Popt} / 1-\operatorname{Popt}\left(\mathrm{r}^{*} \bmod ^{*} 1 /\right.\right.$ Popt $\left.)\right\}$}

Where, $T(S)$ is the threshold, popt is the predetermined percentage of cluster heads, $r$ is the current round.

\subsubsection{Steady State phase}

In steady state phase, the clusters are formed and the corresponding cluster head is selected. After the cluster head receives the data it can be aggregated and the data can be transmitted to the base station. Research on An application-specific protocol architecture for wireless micro sensor networks by W.B.Heinzelman, proposed an solution that, In LEACH-C[4], During the set-up phase each node sends information about its current location to the sink. In order to determining good clusters, the sink needs to ensure that the energy load is evenly distributed among all the nodes. The sink computes average node energy, and determines which nodes have energy below this average. This solution minimize the amount of energy for transmit their data to cluster head. After the cluster head and associated cluster(the node which have energy below the average energy) are found, the sink broadcasts a message that obtains the cluster head ID for each node. If a cluster head ID matches its own ID, that node seems to be an cluster head, otherwise the node determines its TDMA slot for data transmission and goes sleep until it's time to transmit data. Once the cluster heads and associated clusters are found, the sink broadcasts a message that obtains the cluster head ID for each node. If a cluster head ID matches its own ID, the node is a cluster head. Otherwise the node determines its TDMA slot for data transmission and goes sleep until it's time to transmit data.

3.4 Research on Exploiting heterogeneity in sensor networks by M. Yarvis, N.Kushalnagar, H. Singh proposed an solution that,

Use of heterogeneous nodes leads to improve the network reliability and lifetime of the network. Nodes having different energies leads to the selection of clusterhead at regular intervals can be reduced. In Heterogeneous sensor networks, large number of inexpensive nodes performs sensing. While a few expensive nodes provide data filtering, fusion and transport. This partitioning of tasks ensures a cost effective design as well as a more efficient implementation of the overall sensing application.

\section{PROBLEM DEFINITION}

Routing is a challenging task in WSNs because of their unique characteristics which makes it different from other wired and wireless networks like cellular or mobile ad hoc network (MANETs). [6] Due to its deployment nature (large scale deployment), the Internet Protocol (IP) based protocols may not be the better choice to be applied on. In this proposed solution we designed OEECA protocol for selecting Cluster head for forwarding packet to sink node and it consume less energy than LEACH. In this paper, we compare the performance of LEACH [3] and OEECA.In LEACH routing algorithm, re-selection of cluster-head at frequent attempts and frequent dead of cluster-head should be the problem.

\subsection{PROPOSED ALGORITHM-OEECA}

In OEECA approach, sensor nodes form a cluster and in order to send the data to base station cluster- head is selected based on the energy and that sensor nodes is selected as a $\mathrm{CH}$ (cluster-head). While processing of Cluster-head node the energy become reduce, so if the energy of $\mathrm{CH}$ is becomes below the non-cluster-head nodes energies means next round should to be processed. In the next round, the Secondary cluster-head should be made as a lead while selection of cluster-head for the first round, so no need to select the cluster-head for next round. In this case, searching overhead to be minimized. As important that, OEECA approach consumes limited energy to send the data. 


\subsection{OEECA \& LEACH APPROACH}
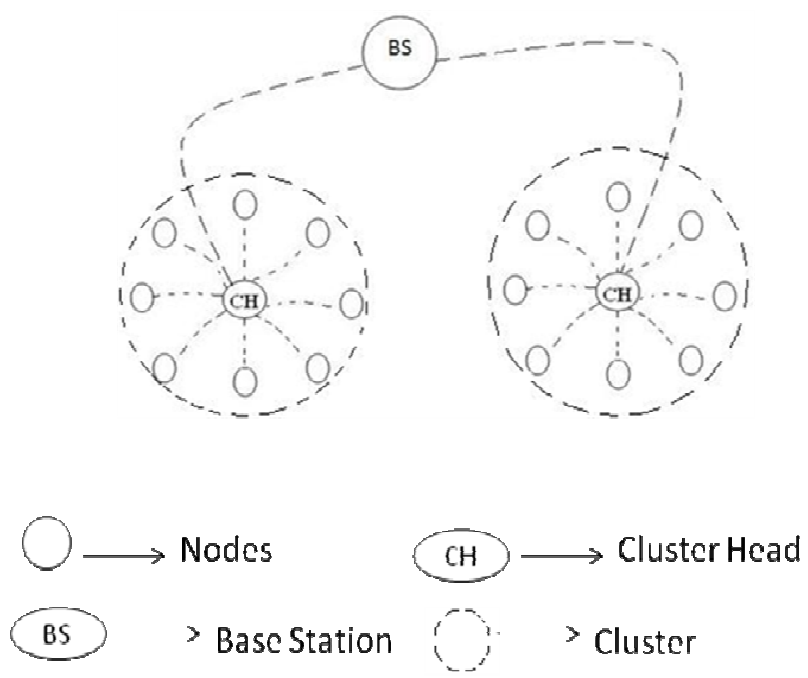

Fig 4.2: Sensor Network Model

\subsection{LEACH}

The main Aim of the LEACH routing protocol is to efficiently maintain the Energy consumption and performing data aggregation inorder to reduce the number transmitted messages to the sink. The operation of LEACH[3] is divided in two phases,

(i) Set-up phase.

(ii) Steady state phase.

\subsubsection{Set-up phase}

In set-up phase, the cluster head is selected and then it forms a group.So after some time the corresponding cluster head energy to be reduced and to rotate the cluster head selection process.

\subsubsection{Steady State phase}

In steady state phase, the groups are formed and the corresponding cluster head is selected. After the cluster head receives the data, it can be aggregated and the data can be transmitted to the base station[2,3].

\subsection{ELIGIBILITY FOR CLUSTER-HEAD}

In order to select the cluster-head, it will satisfy the eligibility criteria. First, average energy to be calculated and then sensor nodes which exceeds energy level than the average energy considered as eligible for Selection of cluster-head.

\subsection{PSEUDOCODE}

\subsubsection{CLUSTER-HEAD SELECTION PROCESS}

During the selection of cluster-head

if

Sensor nodes $>$ than average energy level then

Else

Sensor nodes eligible for cluster-head

Not eligible for Cluster-head selection process Start the process.

\subsubsection{LEACH [3] APPROACH}

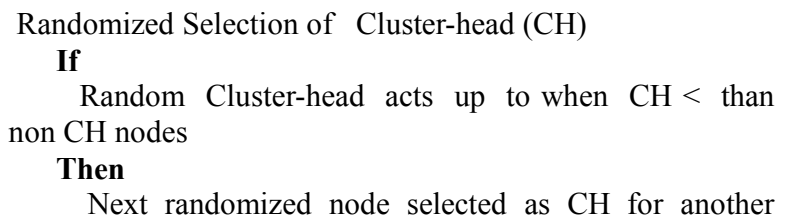
round and the $\mathrm{CH}$

For first round is stored in dummy variable to avoid re-Cluster head selection process.

Else

Already selected $\mathrm{CH}$ to be re-used, for that Else if performance to be reduced

Not eligible for $\mathrm{CH}$ selection process.

\subsubsection{OEECA Approach}

Secondary $\mathrm{CH}$ to be Selected as a Back-end node with the selection of $\mathrm{CH}$ for First round

If

For Selection of Secondary $\mathrm{CH}$, the nodes which have more energy level after the selection of $\mathrm{CH}$ for first round acts as a backend node.

Then

After the reduction in energy level for first round as compare to Average energy, without selecting the $\mathrm{CH}$ for next round the Secondary $\mathrm{CH}$ as a Back-end acts and it can be act for next round.

Else

Reselection of $\mathrm{CH}$ leads to be an selection Over-head.

Else if

More Energy Consumption.

\section{SIMULATION SETUP}

We have used Network Simulator (NS)-2 in our evaluation. The NS-2 is a discrete event driven simulator [11] developed at UC Berkeley. We have used Fedora Linux environment with NS-2 . NS-2 is suitable for designing new protocols, comparing different protocols and traffic evaluations. It is an object oriented simulation written in 
$\mathrm{C}++$, with an $\mathrm{OTcl}$ interpreter as a front-end. Ns-2 Here output screenshot shown in Fig 5.1.

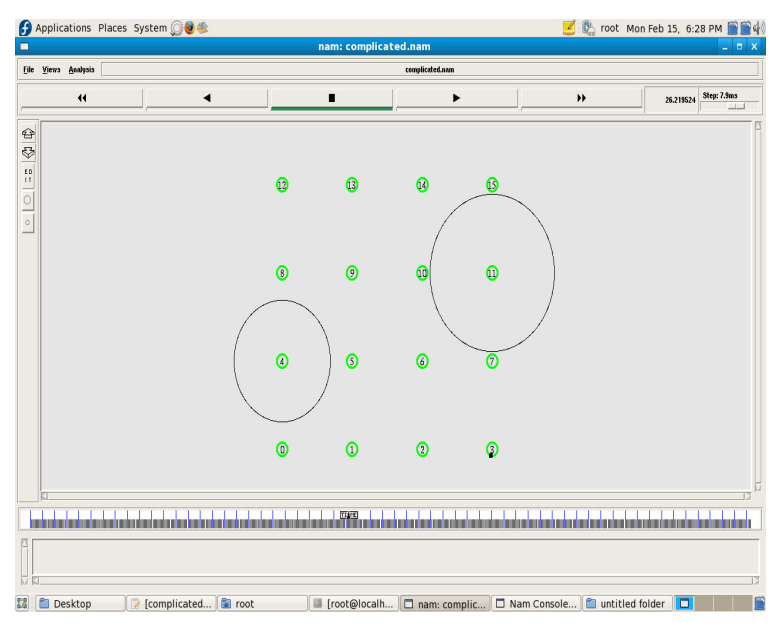

Fig 5.1: System model

\section{PERFORMANCE ANALYSIS}

We have done the simulatation for various scenario and update results in table.Increase in Cluster-head leads to increase in Clusters, reduction in distance between cluster nodes and base station and reduction in energy.

\section{Graph 6.1: OEECA-Cluster head Vs Energy spent}

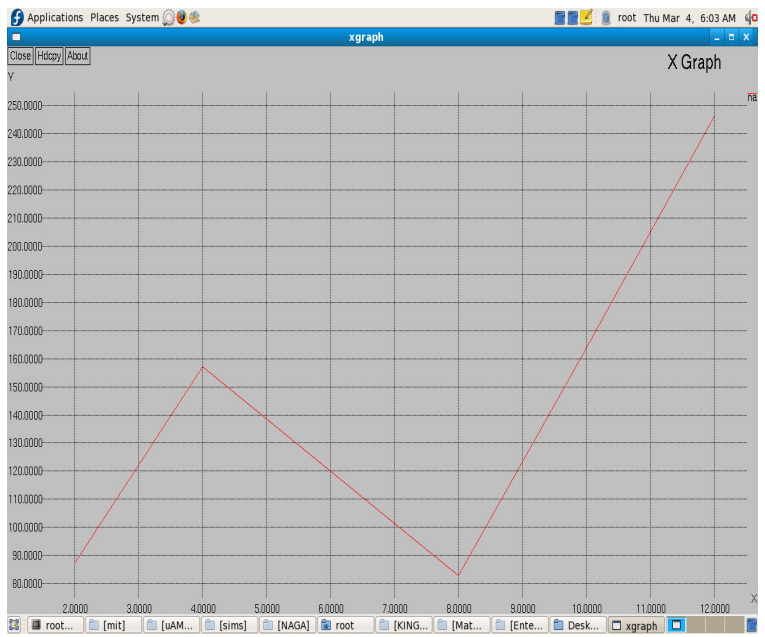

Result analysis shows, increase in simulation time leads to reduction in network life time and increase in dead of cluster-head.

\section{Graph 6.2: OEECA- Time Vs Cluster Node Alive}

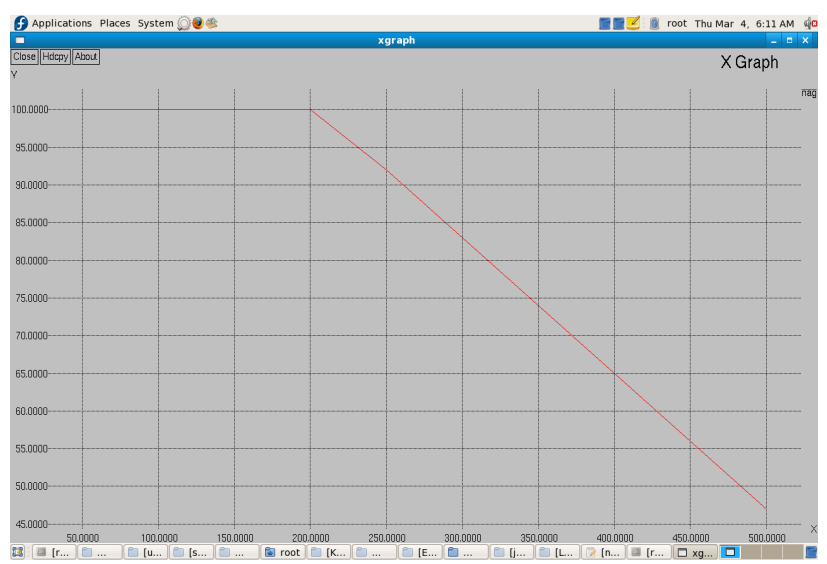

\subsection{LEACH}

Result analysis shows, increase in Clusters leads to more dead in Cluster-head, increase in Energy Consumption and transmission of data to Base-station to be limited.

Tab 6.3: LEACH- No of Clusters vs energy consumed

\begin{tabular}{|c|c|c|}
\hline S.No & $\begin{array}{c}\text { Number of } \\
\text { clusters }\end{array}$ & $\begin{array}{c}\text { Energy } \\
\text { Consumption }\end{array}$ \\
\hline 1 & 2 & 93.25 \\
2 & 6 & 49.64 \\
3 & 10 & 238.57 \\
4 & 18 & 283.217 \\
5 & 24 & 229.73 \\
6 & 26 & 238.677 \\
\hline
\end{tabular}

\section{Graph 6.3.1:LEACH- No of Clusters vs energy consumed}

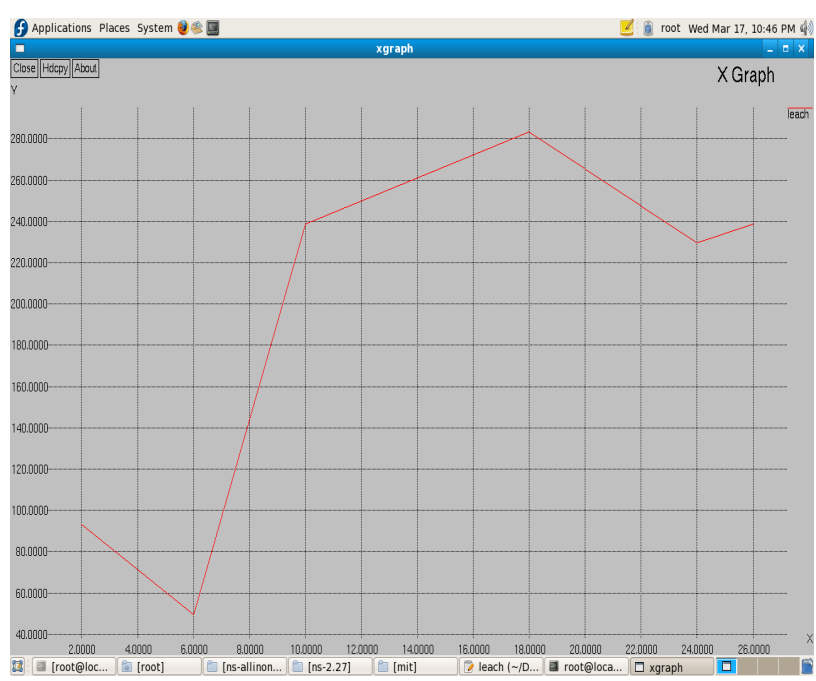




\subsection{OEECA (Proposed Algorithm)}

Result analysis shows, increase in Clusters leads to more dead in Cluster-head, reduction in Energy Consumption and transmission of data to Base-station to be more as compare to LEACH routing Algorithm..

Tab 6.4: OEECA- No of Clusters vs energy consumed

\begin{tabular}{|c|c|c|}
\hline S.No & $\begin{array}{c}\text { Number of } \\
\text { clusters }\end{array}$ & $\begin{array}{c}\text { Energy } \\
\text { Consumption }\end{array}$ \\
\hline & 2 & 20.62 \\
1 & 6 & 45 \\
2 & 10 & 175.49 \\
3 & 18 & 258.17 \\
4 & 24 & 251.07 \\
5 & 26 & 221.07 \\
6 & & \\
& & \\
& & \\
\hline
\end{tabular}

\section{Graph 6.4.1:OEECA- No of Clusters vs energy consumed}

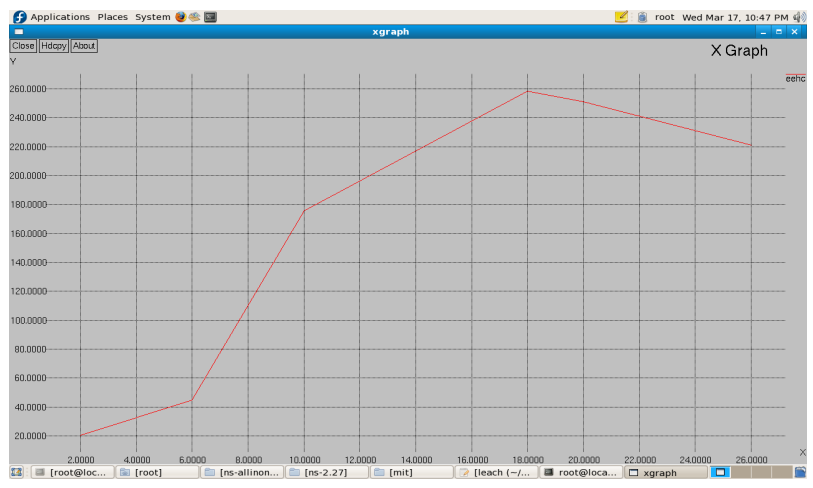

\section{CONCLUSION}

In this paper, we have proposed the Optimal Algorithm for the conservation of energies and inorder to compare the performance for OEECA with the Routing algorithm LEACH.The Results predict that, AS compare to LEACH routing algorithm OEECA algorithm is best in all cases.The performance is based on Energy Consumption, Selection of Cluster-heads,Aggregated data to the base station.

\section{FUTURE WORK}

In Future, the research can be made based on which the OEECA protocol can be compared with all other Routing protocols such as PEGASIS, TEEN,GEAR,SPEED,MMSPEAD .The performance can be analyze in the basis of data transmission with resourceful energy and change of selection of clusterhead can be summarized.

\section{REFERENCES}

[1] K. Khamforoosh, and H. Khamforoush, "A new rounting Algorithm for Energy Reduction in Wireless Sensor Networks", IEEE, 2010

[2] Wendi Heinzelman, Anantha Chandrakasan, and Hari Balakrishnan. Energy- Efficient Communicat ion Protocol for Wireless Microsensor Networks. Proceedings of the Hawaii International Conference on System Sciences, January 4-7, 2000 @ 2000 IEEE.

[3] MIT $\mu$ AMPS project ns2 code extensions.http://wwwmtl.mit.edu/research/icsystems/uamps/research/leach/leach code.shtml

[4] M. Younis, M. Youssef and K. Arisha, "Energy-Aware Routing in Cluster-Based Sensor Networks",in the Proceedings of the 10th IEEE/ACM(MASCOTS2002), Fort Worth, TX, October 2009

[5] C. Prehofer and C. Bettstetter, "Self-organization in communication networks: principles and design paradigms," IEEE Communications Magazine, vol. 43, pp. 78-85, 2009

[6] C. Yongtao and H. Chen, "A distributed clustering algorithm with an adaptive backoff strategy for wireless sensor networks," IEICE Transactions on Communications, vol. E89-B, pp. 609-13, 2008.

[7] Y. Yang, H.-H. Wu, H.-H. Chen, Short: Shortest hop routing tree for wireless sensor networks, in: Proceedings of IEEE ICC-2006, 2006.

[8]. Emad Felemban, C-G Lee. MMSPEED: Multipath Multi-SPEED Protocol for QoS Guarantee of Reliability and Timeliness in Wireless Sensor Networks In IEEE Mobile Computing, vol 5, no 6,June 2006.

[9] Tian He, J A Stankovie. SPEED: A Stateless Protocol for Real- Time Communication in Sensor Networks. 2005

[10] N. Chilamkurti, S. Zeadally, A. Vasilakos, and V. Sharma1, "Cross-Layer Support for Energy Efficient Routing in Wireless Sensor Networks," Journal of Sensors, Hindawi Publishing Corporation, vol. 2009, p. 9 pages, 2009.

[11] Ns-2 network simulator,http://www.isi.edu/nsnam/ns/

[12] J. Malek, "Trace graph - Network Simulator NS-2 trace files analyser," in http://www.tracegraph.com/ 